LAPTH-020/18

\title{
Rotating magnetized black diholes
}

\author{
Gérard Clément* \\ LAPTh, Université Savoie Mont Blanc, CNRS, \\ 9 chemin de Bellevue, BP 110, F-74941 Annecy-le-Vieux cedex, France
}

3 July 2018

\begin{abstract}
We analyze a four-parameter class of asymptotically flat magnetized solutions to the Einstein-Maxwell equations constructed by Manko et al., and show that these represent systems of two co-rotating extreme black holes with equal masses and electric charges, and opposite magnetic and NUT charges, connected by a cosmic string. We discuss several three-parameter subclasses, and determine in each case the parameter domain in which the ring singularity is absent. We find a two-parameter subclass and a one-parameter subclass where the conical singularity is also absent in the horizon co-rotating frame.
\end{abstract}

*Email: gclement@lapth.cnrs.fr 


\section{Introduction}

Since the early days of general relativity, it has been known that axisymmetric multi-black hole solutions to the Einstein equations can be constructed from linear superpositions of one-black hole solutions [1]. These generically present conical singularities 2] on the symmetry axis (cosmic strings) to account for the forces necessary to balance the attraction between black holes, the only known exception being the static Majumdar-Papapetrou [3] linear superposition of identical extreme Reissner-Nordström black holes.

The existence of more general, non-linear multi-black hole solutions to the Einstein or Einstein-Maxwell equations has been proved in the harmonic map analysis of [4]. A number of such rotating solutions have been constructed by inverse scattering techniques, see the review in [5], the most recent work on the subject being probably [6] on the two-Kerr system. However little is generally known about their structure. It was first shown by Emparan [7] that the static magnetized Bonnor solution [8] actually represents a black dihole, a system of two black holes with opposite magnetic charges and degenerate horizons, held apart by a cosmic string. This string can be removed by applying an external magnetic field, at the expense of asymptotic flatness. Recently, we have shown [9] that a rotating solution to the Einstein-Maxwell equations previously constructed in [10] represents a more complex system of two extreme black holes with equal masses and electric charges, and opposite magnetic and gravimagnetic (NUT) charges, co-rotating at an angular velocity fine-tuned to one-fourth of the inverse NUT charge. A third, necessary partner in this system is an electrically charged, magnetized strut or string which also acts as a Dirac-Misner string.

Both the Bonnor solution and the solution studied in 9] belong to a larger four-parameter class of solutions constructed with the aid of Sibgatullin's method [11] by Manko et al. in [12], where their connection with neutron star models was suggested. Perhaps because of their complexity, the properties of these solutions have not, to our knowledge, been investigated. We will show in the present paper that all these solutions also represent co-rotating magnetized black diholes, with characteristics similar to those elucidated in [9]: equal masses and electric charges, opposite magnetic and NUT charges, and the existence of a cosmic-Dirac-Misner string connecting the two degenerate horizons. For several three-parameter subclasses, we will discuss the algebraic conditions for the absence of ring singularities - a necessary condition for the global solution to be quasi-regular, with only a mild conical singularity. Interestingly, we shall also find a two-parameter subclass and a one-parameter subclass where the conical singularity itself is absent in 
the horizon co-rotating frame, leading to apparently regular two-black-hole solutions.

The solutions of [12] are presented in the next section. In section 3 we discuss the properties of the horizons and compute the various horizon charges. The interconnecting string is considered in section 4. Several threeparameter subclasses are further discussed in the next three sections, and our conclusions are presented in the last section.

\section{The solutions}

\subsection{General form}

Let us start from Manko et al's four-parameter asymptotically flat rotating magnetized solution [12] (a subfamily of the nine-parameter electrovac solutions of [13]). In a first step, we will choose as independent parameters the overall scale $\kappa>0$, and three dimensionless parameters $m>0, a$ and $b$ which are those of Manko et al. (here indexed with $M$ ) divided by $\kappa$ (noted $k$ in [12]), i.e. $m=M_{M} / \kappa, a=a_{M} / \kappa, b=b_{M} / \kappa$. Auxiliary parameters are $d=d_{M} / \kappa^{2}, \delta=\delta_{M} / \kappa^{2}$ and $\bar{\mu}=\mu_{M} / \kappa^{2}$ defined by

$$
4 d=m^{2}-(a-b)^{2}, \quad \delta=1-d, \quad \bar{\mu}^{2}=m^{2} b^{2}+4 d \delta .
$$

The physical parameters, total mass $M$, total angular momentum $J$ and total dipole magnetic moment $\mu$ are related to the preceding by

$$
M=\kappa m, \quad J=\kappa^{2} m a, \quad \mu=\kappa^{2} \bar{\mu} .
$$

The Ernst potentials $1 \mathcal{E}, \psi$ may be expressed in terms of Kinnersley potentials $(U, V, W)$ according to

$$
(1970) \mathcal{E}=(U-W) /(U+W), \quad \psi=V /(U+W),
$$

The Kinnersley potentials for the Manko et al. family of solutions (labelled $A^{*} / 4 \kappa^{4}, i \mu C^{*} / 2 \kappa^{4}, m B^{*} / 2 \kappa^{4}$ in [12], where $*$ denotes the complex conjugate) are given in prolate spheroidal coordinates by:

$$
\begin{aligned}
U= & \left(x^{2}-\delta y^{2}\right)^{2}-d^{2}-\nu \lambda\left(1-y^{4}\right)+2 i x y\left[\nu\left(x^{2}-1\right)+\lambda\left(1-y^{2}\right)\right] \\
V= & \bar{\mu}\left\{-\nu x\left(1-y^{2}\right)+i y\left[\left(x^{2}-1\right)+\delta\left(1-y^{2}\right)\right]\right\} \\
W= & m x\left[\left(x^{2}-1\right)+(b \nu+\delta)\left(1-y^{2}\right)\right]- \\
& -i m y\left[b\left(x^{2}-1\right)+(b \delta-\lambda)\left(1-y^{2}\right)\right] .
\end{aligned}
$$

\footnotetext{
${ }^{1}$ We use the same conventions for defining the Ernst potentials as in 14, equations (3.1)-(3.3).
} 
The prolate spheroidal coordinates $x \geq 1, y \in[-1,+1]$ are related to the Weyl cylindrical coordinates $\rho, z$ by

$$
\rho=\kappa \sqrt{\left(x^{2}-1\right)\left(1-y^{2}\right)}, \quad z=\kappa x y .
$$

To simplify the form of the solution, we have introduced two new dimensionless parameters $\nu$ and $\lambda$ which play symmetrical roles in (2.4). These are related to our original parameters by

$$
\nu \equiv(a-b) / 2, \quad \lambda \equiv \nu(d-\delta)-m^{2} b / 2 .
$$

The other dimensionless parameters occurring in (2.4) are related to $m, \nu$ and $\lambda$ by

$$
\delta=1+\nu^{2}-\frac{m^{2}}{4}, \quad d=1-\delta, \quad b=\nu-\frac{4}{m^{2}}\left[\nu^{3}+\frac{\nu+\lambda}{2}\right],
$$

$\bar{\mu}$ being given in terms of these by the last equation (2.1) 2. We note also that, from (2.4), the total quadrupole electric moment is $Q_{2}=-\kappa^{3} \bar{\mu} \nu$, so that $\nu$ is a measure of the electric quadrupole to magnetic dipole ratio.

The corresponding metric and electromagnetic potential may be written in the generic form

$$
\begin{aligned}
d s^{2} & =-\frac{f}{\Sigma}\left(d t-\frac{\kappa \Pi}{f} d \varphi\right)^{2}+ \\
& +\kappa^{2} \Sigma\left[\left(x^{2}-y^{2}\right)^{-3}\left(\frac{d x^{2}}{x^{2}-1}+\frac{d y^{2}}{1-y^{2}}\right)+f^{-1}\left(x^{2}-1\right)\left(1-y^{2}\right) d \varphi^{2}\right] \\
A & =\frac{1}{\Sigma}[\bar{v} d t+\kappa \Theta d \varphi],
\end{aligned}
$$

where the various functions, evaluated in [12, are

$$
\begin{aligned}
f(x, y) & =\left[\zeta^{2}+\nu \lambda\left(1-y^{2}\right)^{2}\right]^{2}-4\left(x^{2}-1\right)\left(1-y^{2}\right)\left(\nu x^{2}-\lambda y^{2}\right)^{2} \\
\Sigma(x, y) & =\left\{\zeta(\zeta+m x+2 d)+m b \nu x\left(1-y^{2}\right)-\lambda \nu\left(1-y^{4}\right)\right\}^{2}+ \\
& +y^{2}\left\{2 x\left[\nu\left(x^{2}-1\right)+\lambda\left(1-y^{2}\right)\right]+m\left[-b \zeta+\lambda\left(1-y^{2}\right)\right]\right\}^{2}, \\
\Pi(x, y) & =-\left(1-y^{2}\right)\left\{( x ^ { 2 } - 1 ) ( \nu x ^ { 2 } - \lambda y ^ { 2 } ) \left(4 m x\left[\zeta+m x+2 d-b \nu\left(1+y^{2}\right)\right]+\right.\right. \\
& \left.+2\left(m^{2} b^{2}-4 d \delta\right) y^{2}\right)+\left[\zeta^{2}+\nu \lambda\left(1-y^{2}\right)^{2}\right] .
\end{aligned}
$$

\footnotetext{
${ }^{2}$ Note that the reality of $\bar{\mu}$ is ensured only in a sector of the three-space $(m, \nu, \lambda)$.
} 


$$
\begin{aligned}
& \left.\cdot\left(2 m b(x+m) \zeta+\left[-m \lambda(2 x+m)+\frac{\nu}{2}\left(m^{2} b^{2}-4 d \delta\right)\right]\left(1-y^{2}\right)\right)\right\}, \\
\bar{v}(x, y)= & \bar{\mu}\left\{-\nu x\left(1-y^{2}\right)\left(\zeta(\zeta+m x+2 d)+m b \nu x\left(1-y^{2}\right)-\lambda \nu\left(1-y^{4}\right)\right)+\right. \\
& \left.+y^{2} \zeta\left(2 x\left[\nu\left(x^{2}-1\right)+\lambda\left(1-y^{2}\right)\right]+m\left[-b \zeta+\lambda\left(1-y^{2}\right)\right]\right)\right\} \\
\Theta(x, y)= & \frac{\bar{\mu}\left(1-y^{2}\right)}{2}\left\{\left[\zeta(\zeta+m x+2 d)+m b \nu x\left(1-y^{2}\right)-\lambda \nu\left(1-y^{4}\right)\right] .\right. \\
\cdot & {\left.\left[(2 x+m)\left(\zeta+m^{2}\right)+2 m\left(x^{2}-d-2 \nu^{2}\right)\right)-m b \nu\left(1+y^{2}\right)\right]+ } \\
& +2 y^{2}\left[2 x\left(\nu\left(x^{2}-1\right)+\lambda\left(1-y^{2}\right)\right)+m\left(-b \zeta+\lambda\left(1-y^{2}\right)\right)\right] . \\
& \left.\left.\cdot\left[m b(x+m)-\nu x^{2}+\lambda\right)\right]\right\} .
\end{aligned}
$$

In the preceding, we have put

$$
\zeta \equiv x^{2}-1+\delta\left(1-y^{2}\right)
$$

The angular variable $\varphi$ is assumed to have the standard periodicity $2 \pi$, so that the metric is asymptotically flat.

In the special case $\delta=\lambda=0$, we recover the special rotating dihole solution analyzed in [9]. We shall show in the following that the more general solution discussed here shares similar properties. The metric (2.8) describes a system of two co-rotating black holes of horizons $x=1, y= \pm 1$ (discussed in section 3), connected by a string (a segment along which the metric has a conical singularity) $x=1, y^{2}<1$ (discused in section 4 ). There is also an ergosphere, where $f(x, y)<0$, generically bounded by two surfaces connecting the two ends of the string ( $f$ is positive on the string, except if $\left.\delta^{2}+\nu \lambda=0\right)$. The two black holes generically have equal masses and electric charges, and opposite NUT charges and magnetic charges. This metric can also present a naked ring singularity, which is the locus where the function $U+W$ vanishes. As opposed to the conical singularity of the string, this is a strong curvature singularity, so only the solutions where this ring singularity is absent can be considered as quasi-regular.

\subsection{Special cases}

Although with our parameterization the general form (2.4) of the Kinnersley potentials looks rather simple, the discussion of the physical properties turns out to be very intricate in the general four-parameter case, due to the non-linear dependence of the auxiliary parameters $\delta, d, b$, and $\bar{\mu}$ on the

dimensionless parameters $m, \nu$ and $\lambda$, and the additional constraint on the 
reality of $\bar{\mu}$. Especially, the absence of ring singularity is technically difficult to prove (or disprove) in the general case. In the present paper, we will only discuss this question in the case of several special three- or two-parameter sub-spaces.

a) $\underline{\delta=\lambda=0}$. This class of solutions depending only on two parameters, e.g. the total mass and the total angular momentum, is a magnetized rotating generalization of the " $\delta=2$ " 3 static Zipoy-Voorhees [15] vacuum solution, different from the " $\delta=2$ " rotating Tomimatsu-Sato [16] vacuum solution (TS2). These solutions were discussed in detail in [9], the parameters introduced in the present paper being related to those of [9] by

$$
m=\frac{2}{p}, \quad \nu=\frac{q}{p}, \quad b=\frac{p q}{2}, \quad \bar{\mu}=-\varepsilon q
$$

$\left(q^{2}+p^{2}=1\right)$. Remarkably, all the solutions of this class are free from a naked ring singularity. We will not discuss further these solutions here.

b) $2 m+d=0$. The solutions of this three-parameter class, analyzed in section 5, have electrically neutral constituents (horizons and string). We will show that the ring singularity is absent in two disjoint three-parameter subsectors.

c) $\underline{\nu=0}$. This constraint corresponds to the vanishing of the total electric quadrupole moment $Q_{2}$, resulting from a delicate balance between electrically charged horizons and string. This three-parameter class is discussed in section 6 , where we show that the ring singularity is absent in the sector $m<2$. We also single out two two-parameter subclasses. The first subclass $b=0(\lambda=0)$ coincides with the static Bonnor solution [8], while the second subclass $b=b_{c}(m)$ (given in (6.8) ) is characterized by a vanishing string tension in the horizon co-rotating frame.

d) $\underline{\lambda=\nu}$. This three-parameter class, characterized by a vanishing horizon angular velocity is discussed in section 7, where we identify a ring singularity-free sector (equation (7.8)). Three special two-parameter subclasses are $\nu=0$ (the Bonnor solution), $\delta=0$ (the TS2 solution), and $\delta=m^{2} / 2\left(\nu^{2}=3 m^{2} / 4-1\right)$, leading to a vanishing total angular momentum. This last subclass contains a one-parameter family $m=m_{c} \simeq 1.30$ with again a vanishing string tension.

Before analyzing these various special cases, we discuss in the next two sections the general physical properties of the two-component horizon and of the interconnecting string.

\footnotetext{
${ }^{3}$ This parameter $\delta$ has no relation with the parameter $\delta$ used in the present paper.
} 


\section{Horizons}

The "points" $x=1, y= \pm 1(\rho=0, z= \pm \kappa)$ are actually horizons, twosurfaces with a finite area which shall be computed below. To see this, take the limit $x \rightarrow 1$ and $y \rightarrow \pm 1$ with the ratio

$$
X^{2}=\frac{1-y^{2}}{x^{2}-1}
$$

held fixed. In this limit, the reduced (barred) Kinnersley potentials defined by $(U, V, W)=(\bar{U}, \bar{V}, \bar{W})\left(x^{2}-1\right)$ go to

$$
\begin{aligned}
\bar{U} & =2\left\{d+(d \delta-\nu \lambda) X^{2} \pm i\left(\nu+\lambda X^{2}\right)\right\}, \\
\bar{V} & =\bar{\mu}\left\{\nu X^{2} \pm i\left(1+\delta X^{2}\right)\right\}, \\
\bar{W} & =m\left\{1+(b \nu+\delta) X^{2} \mp i\left[b+(b \delta-\lambda) X^{2}\right]\right\} .
\end{aligned}
$$

In the same limit, the auxiliary function $\zeta(x, y)$ behaves as $\zeta \sim\left[1+\delta X^{2}\right]\left(x^{2}-\right.$ 1), leading for $\lambda \neq \nu$ to

$$
\begin{aligned}
f & \sim-4(\nu-\lambda)^{2} X^{2}\left(x^{2}-1\right)^{2} \\
\Sigma & \sim \Sigma(X)\left(x^{2}-1\right)^{2} \\
\Pi & \sim 2(\lambda-\nu)\left[(m b-2 \nu)^{2}+(m+2 d)^{2}\right] X^{2}\left(x^{2}-1\right)^{2}
\end{aligned}
$$

where

$$
\begin{aligned}
\bar{\Sigma}(X) & =\left(m+2 d+[(m+2 d) \delta+(m b-2 \lambda) \nu] X^{2}\right)^{2}+ \\
& +\left(2 \nu-m b+[(m+2 d) \lambda-(m b-2 \lambda) \delta] X^{2}\right)^{2} .
\end{aligned}
$$

It follows that

$$
g_{\varphi \varphi}=\kappa^{2}\left[\frac{\Sigma}{f}\left(x^{2}-1\right)\left(1-y^{2}\right)-\frac{\Pi^{2}}{\Sigma f}\right] \sim-\frac{\kappa^{2} \Pi^{2}}{\Sigma f}
$$

is finite and positive, while the lapse

$$
N^{2}=\frac{\kappa^{2}\left(x^{2}-1\right)\left(1-y^{2}\right)}{g_{\varphi \varphi}}
$$

develops a double zero at $x=1, y= \pm 1$, corresponding to two double horizons $H_{ \pm}$, co-rotating at the angular velocity

$$
\Omega_{H}=\left.\frac{f}{\kappa \Pi}\right|_{H}=\frac{2(\nu-\lambda)}{\kappa\left[(m+2 d)^{2}+(m b-2 \nu)^{2}\right]} .
$$


Let us transform from $(x, y)$ to the coordinates [17] $X$ and $Y=y / x$. Noting that, near the horizons $Y= \pm 1$,

$$
-\frac{\Pi^{2}}{f} \sim \bar{\Sigma}_{0}^{2} X^{2}\left(x^{2}-1\right)^{2}
$$

where $\bar{\Sigma}_{0} \equiv \bar{\Sigma}(0)$, one finds that the horizon metric degenerates to

$$
d s_{H}^{2}=\kappa^{2} \bar{\Sigma}(X)\left[\frac{d X^{2}}{\left(X^{2}+1\right)^{4}}+\left(\frac{\bar{\Sigma}_{0}}{\bar{\Sigma}(X)}\right)^{2} X^{2} d \hat{\varphi}^{2}\right]
$$

in the co-rotating near-horizon frame $(\hat{t}, X, Y, \hat{\varphi})$ defined by $\hat{t}=t, \hat{\varphi}=$ $\varphi-\Omega_{H} t$. The horizon area is

$$
\mathcal{A}_{H}=\pi \kappa^{2} \bar{\Sigma}_{0}=\pi \kappa^{2}\left[(m+2 d)^{2}+(m b-2 \nu)^{2}\right] .
$$

The horizon metric (3.9) is regular at $X=0(y= \pm 1)$, but generically presents a conical singularity at $X \rightarrow \infty(x=1)$ with deficit angle $2 \pi(1-$ $\left.\alpha_{H}\right)$, where

$$
\alpha_{H}=\frac{\bar{\Sigma}_{0}}{\bar{\Sigma}_{4}}
$$

with

$$
\bar{\Sigma}_{4}=[(m+2 d) \delta+(m b-2 \lambda) \nu]^{2}+[(m+2 d) \lambda-(m b-2 \lambda) \delta]^{2}
$$

the coefficient of $X^{4}$ in $\bar{\Sigma}(X)$. Again, it is difficult to determinate the sign of this deficit angle in the general case.

The electromagnetic potential on the horizon is, in the co-rotating frame,

$$
\begin{aligned}
\hat{A}= & -\frac{\bar{\mu}(m b-2 \nu)}{\left[(m b-2 \nu)^{2}+(m+2 d)^{2}\right]} d t+\frac{\kappa \bar{\Theta}(X)}{\bar{\Sigma}(X)} d \hat{\varphi}, \\
\bar{\Theta}= & \frac{\bar{\mu}}{2} X^{2}\left\{((m+2)(m+2 d)-2 \nu(m b-2 \nu))\left(m+2 d+[(m+2 d) \delta+(m b-2 \lambda) \nu] X^{2}\right)+\right. \\
& \left.+2(m b(m+1)+\lambda-\nu)\left(2 \nu-m b+[(m+2 d) \lambda-(m b-2 \lambda) \delta] X^{2}\right)\right\} .
\end{aligned}
$$

The horizon vector potential generates a magnetic field perpendicular to the horizon, flux conservation implying that the two horizons carry opposite magnetic charges $\pm P_{H}$, with

$$
P_{H}=-\frac{1}{4 \pi} \oint_{H_{+}} d A_{\varphi} d \varphi=-\left.\frac{1}{2} A_{\varphi}(X)\right|_{\infty} ^{0}=\frac{\kappa \bar{\Theta}_{4}}{2 \bar{\Sigma}_{4}},
$$


where $\bar{\Theta}_{4}$ is the coefficient of $X^{4}$ in $\bar{\Theta}(X)$.

Computation of the horizon electric charges, which are equal because the electric potential is even in $y$, necessitates in principle the knowledge of the electric field off the horizon. However we can use for this purpose the Tomimatsu formula [18], which necessitates only the knowledge of the Kinnersley potentials on the horizon and that of the horizon angular velocity:

$$
Q_{H}=\frac{1}{4 \pi} \oint_{H_{+}} \omega \frac{d \operatorname{Im} \psi}{d X} d X d \varphi=\left.\frac{\omega_{H}}{2} \operatorname{Im} \psi(X)\right|_{\infty} ^{0}
$$

with $\omega_{H}=1 / \Omega_{H}$ the constant value of $\omega=\kappa \Pi / f$ over the horizon, and

$$
\operatorname{Im} \psi=\frac{\operatorname{Re}(\bar{U}+\bar{W}) \operatorname{Im} \bar{V}-\operatorname{Re} \bar{V} \operatorname{Im}(\bar{U}+\bar{W})}{|\bar{U}+\bar{W}|^{2}} .
$$

The result is

$$
Q_{H}=\frac{\kappa \bar{\mu}(m+2 d)}{4 \bar{\Sigma}_{4}}\left[\nu m^{2} b^{2}-4 \nu\left(\delta^{2}+\nu \lambda\right)+2 \delta m b(m+2)-\lambda(m+2)^{2}\right] .
$$

The horizon Komar mass and angular momentum are given by the Tomimatsu formulas [18] (as corrected in [14]), written here for degenerate black holes:

$$
\begin{aligned}
M_{H} & =\frac{1}{8 \pi} \oint_{H}\left[\omega d \operatorname{Im} \mathcal{E}+2 d\left(A_{\varphi} \operatorname{Im} \psi\right)\right] d \varphi \\
J_{H} & =\frac{1}{8 \pi} \oint_{H} \omega\left[\frac{1}{2} \omega d \operatorname{Im} \mathcal{E}+d\left(A_{\varphi} \operatorname{Im} \psi\right)+\omega \hat{A}_{t} d \operatorname{Im} \psi\right] d \varphi
\end{aligned}
$$

The computation gives, after some simplifications,

$$
\begin{aligned}
M_{H}= & \frac{\kappa m}{2} \frac{1}{\bar{\Sigma}_{4}}\left[\left(\delta^{2}-b d \lambda\right)(m+2 d)^{2}+2 \delta \gamma(b d+\nu)(m+2 d)+\right. \\
& \left.+2 b d \delta^{2}(\beta+\gamma)+\left(\delta^{2}+b d \nu\right)\left(m^{2} b^{2}-4 \lambda \nu\right)+m b \nu(\beta \lambda+\gamma \nu)\right]- \\
& -\frac{\bar{\mu}(m+2 d)\left(\delta^{2}+\nu \lambda\right) P_{H}}{\bar{\Sigma}_{4}}
\end{aligned}
$$

where we have put

$$
\beta \equiv m b-2 \nu, \quad \gamma \equiv m b-2 \lambda .
$$

The second term is the contribution of the second piece of (3.18), which was missing in the original Tomimatsu formula. This term, proportional 
to the product of the horizon electric and magnetic charges, vanishes for $m+2 d=0$, as well as for $\delta=\lambda=0$ (the case studied in [9]). As shown in [14], the system of (3.19), (3.18) and (3.15) leads to the horizon Smarr formula for degenerate black holes

$$
M_{H}=2 \Omega_{H} J_{H}+\Phi_{H} Q_{H},
$$

with $-\Phi_{H}=\hat{A}_{t}$ the horizon electric potential in the co-rotating frame.

\section{String}

The string is the coordinate singularity $x=1, y^{2}<1(\rho=0,-\kappa<z<\kappa)$. Near the string, i.e. for $\xi^{2} \equiv x^{2}-1 \rightarrow 0$, using $\zeta=\delta\left(1-y^{2}\right)+\xi^{2}$, we obtain

$$
\begin{aligned}
& f \simeq f_{S}=\left(\delta^{2}+\nu \lambda\right)^{2}\left(1-y^{2}\right)^{4} \quad \text { if } \quad \delta^{2}+\nu \lambda \neq 0, \\
& f \simeq f_{C}=-4\left(1-y^{2}\right)\left(\nu-\lambda y^{2}\right)^{2} \xi^{2} \quad \text { if } \quad \delta^{2}+\nu \lambda=0 .
\end{aligned}
$$

This second case is that of a spinning cosmic string (in a background curved spacetime), with spin $\Omega_{H}^{-1} / 4$, rotating at the critical angular velocity $\Omega_{H}$. We will not treat here this case, similar to that considered in [9], and discuss only the case $\delta^{2}+\nu \lambda \neq 0$.

In this case,

$$
\begin{aligned}
\Sigma_{S} \simeq & \left\{\left[(m b-2 \lambda) \nu+(m+2 d) \delta+\left(\delta^{2}+\nu \lambda\right)\left(1-y^{2}\right)\right]^{2}+\right. \\
& \left.+[(m b-2 \lambda) \delta-(m+2 d) \lambda]^{2} y^{2}\right\}\left(1-y^{2}\right)^{2} \\
\Pi_{S} \simeq & -\left(\delta^{2}+\nu \lambda\right)\left[2 m b(m+1) \delta-m(m+2) \lambda+\frac{\nu}{2}\left(m^{2} b^{2}-4 d \delta\right)\right]\left(1-y^{2}\right)^{4},
\end{aligned}
$$

and the near-string metric is

$$
d s^{2} \simeq-F_{S}\left(d t-\omega_{S} d \varphi\right)^{2}+\frac{\kappa^{2} \Sigma_{S}}{\left(1-y^{2}\right)^{3}}\left[\frac{d y^{2}}{1-y^{2}}+d \xi^{2}+\alpha_{S}^{2} \xi^{2} d \varphi^{2}\right]
$$

where $F_{S}=f_{S} / \Sigma_{S}, \omega_{S}=\kappa \Pi_{S} / f_{S}$ is a constant, and

$$
\alpha_{S}=\frac{1}{\delta^{2}+\nu \lambda}
$$

What is relevant for comparison with the horizon metric (3.9) is the near-string metric in the horizon co-rotating frame $\left(d \varphi=d \hat{\varphi}+\Omega_{H} d t\right)$,

$$
d s^{2} \simeq-\hat{F}_{S}\left(d t-\hat{\omega}_{S} d \hat{\varphi}\right)^{2}+\frac{\kappa^{2} \Sigma_{S}}{\left(1-y^{2}\right)^{3}}\left[\frac{d y^{2}}{1-y^{2}}+d \xi^{2}+\hat{\alpha}_{S}^{2} \xi^{2} d \hat{\varphi}^{2}\right]
$$


where

$$
\hat{F}_{S}=\left(1-\Omega_{H} \omega_{S}\right)^{2} F, \quad \hat{\omega}_{S}=\frac{\omega_{S}}{\left(1-\Omega_{H} \omega_{S}\right)}, \quad \hat{\alpha}_{S}=\frac{\alpha_{S}}{\left(1-\Omega_{H} \omega_{S}\right)} .
$$

A lengthy computation yields, after simplification,

$$
\hat{\alpha}_{S}=\alpha_{H}
$$

where $\alpha_{H}$ is given by (3.11), i.e. the string and horizon conical singularities are equal, when computed in the same reference frame.

As shown in [9], the spin of the spinning cosmic string metric (4.6) should actually be interpreted as a gravimagnetic flow along the Misner string connecting two NUT sources at $\rho=0, z= \pm \kappa$, with NUT charges $\pm N_{H}$,

$$
N_{H}=-\frac{\hat{\omega}_{S}}{4}=\frac{\alpha_{S}-\alpha_{H}}{4 \Omega_{H} \alpha_{S}} .
$$

One obtains

$N_{H}=\frac{\kappa \alpha_{H}}{8}\left[4 \delta^{2}(m b-\lambda-\nu)+\nu\left(m^{2} b^{2}-4 \lambda \nu\right)+2 \delta(m+2 d)(m b-2 \lambda)-\lambda(m+2 d)^{2}\right]$.

This value, as well as those of the other black hole observables, reduce to those given in 9 for $\delta=\lambda=0$.

\section{The neutral class of solutions}

Equation (3.17) shows that the horizon electric charge vanishes for

$$
m+2 d=0 .
$$

Because the global electric charge has been set to zero, the string electric charge will also vanish. We will refer to the class $(m+2 d=0)$ of solutions with electrically neutral constituents as the neutral class.

The constraint (5.1) leaves only the two independent dimensionless parameters $m$ and $b$, the other parameters being related to these by

$$
\delta=\frac{m+2}{2}, \nu^{2}=\frac{m(m+2)}{4}, \lambda=-\left[(m+1) \nu+\frac{m^{2} b}{2}\right], \bar{\mu}^{2}=m^{2} b^{2}-4 \nu^{2},
$$

this last relation implying the restriction

$$
b^{2}>\frac{m+2}{m} .
$$




\subsection{Absence of ring singularity}

We wish to further constrain the parameters in this class so that the ring singularity is absent. The ring singularity corresponds to a zero of $\Sigma(x, y)$, i.e. to a solution of the system

$$
\operatorname{Re}(U+W)=0, \quad \operatorname{Im}(U+W)=0
$$

with $y^{2} \neq 1$. The first equation (5.4) reads, for $m+2 d=0$,

$$
\zeta[\zeta+m(x-1)]+(m+1) \nu^{2}\left(1-y^{2}\right)+m b \nu\left[x+\frac{m\left(1+y^{2}\right)}{2}\right]\left(1-y^{2}\right)=0 .
$$

With $x \geq 1, y^{2}<1$ and $m>0, \delta>0$, a sufficient condition for the absence of solutions to this equation, and thus absence of ring singularity, is obviously $b \nu \geq 0$, implying on account of (5.2) and (5.3) $b \nu>(m+2) / 2$, or $m b / 2 \nu>1$.

The second equation (5.4) is trivially satisfied in the equatorial plane $y=0$, in which case the first equation reduces to the quartic equation in $x$ :

$$
x^{4}+m x^{3}+m(b \nu-d) x-\left(d^{2}+\nu \lambda\right)=0 .
$$

It is clear that for $b \nu<0$ and large enough this equation will have a solution for some $x>1$. For $x=1, m+2 d=0$, the equation reduces to

$$
2 m b \nu+m(m+2)+2=0,
$$

so that a necessary condition for the equatorial ring singularity to be absent is

$$
2 m b \nu+m(m+2)>-2, \quad \text { or } \quad \frac{m b}{2 \nu}>-1-\frac{2}{m(m+2)} .
$$

If $y \neq 0,1-y^{2}$ can be eliminated between the two equations (5.4), leading to an equation of sixth degree in $x$. It is not clear whether this equation can have a solution for $m b / 2 \nu<-1$, and in that case whether such solutions (non-equatorial ring singularities) could be excluded for some range of parameter values.

To conclude this subsection, the neutral solution is certainly free from a ring singularity in the sector $m b / 2 \nu>1$, and possibly free from a ring singularity in the distinct sector $-1-2 / m(m+2)<m b / 2 \nu<-1$. We will see in the following that the properties of the two-black hole system in these two sectors are quite different. 


\subsection{Black hole properties}

The values of the various horizon parameters computed in the general case simplify in this case to

$$
\begin{aligned}
\Omega_{H} & =\frac{2(\nu-\lambda)}{\kappa(m b-2 \nu)^{2}}, \quad \alpha_{H}=\frac{1}{\delta^{2}+\nu^{2}}\left(\frac{m b-2 \nu}{m b-2 \lambda}\right)^{2}, \\
M_{H} & =-\frac{\kappa m(m b-2 \nu)\left(\delta^{2}+m b \nu / 2\right)}{2(m b-2 \lambda)\left(\delta^{2}+\nu^{2}\right)}, \\
N_{H} & =\frac{\kappa \alpha_{H}}{8}\left[4\left(\delta^{2}+\nu^{2}\right)(m b-\lambda-\nu)+\nu(m b-2 \nu)^{2}\right], \\
Q_{H} & =0, \quad P_{H}=-\frac{\kappa \bar{\mu}(m b-2 \nu)}{2(m b-2 \lambda)},
\end{aligned}
$$

and the horizon angular momentum

$$
J_{H}=\frac{M_{H}}{2 \Omega_{H}}
$$

from the Smarr formula for an electrically neutral degenerate black hole.

It follows from the last equation (5.2) that, for a vacuum solution $(\bar{\mu}=0)$, either one of the two combinations $(m b-2 \nu)$ and

$$
(m b-2 \lambda)=(m+1)(m b+2 \nu)
$$

must vanish, with unpleasant consequences (for $m b-2 \nu=0$ the horizon area (3.10) vanishes, while for $m b-2 \lambda=0$, the angular deficit and the horizon Komar mass diverge). On the other hand, for a non-vacuum solution $(\bar{\mu} \neq 0)$, the combinations $(m b-2 \nu)$ and $(m b-2 \lambda)$ do not vanish and are of the same sign. It then follows from the regularity condition (5.8), which implies $\delta^{2}+m b \nu / 2>(m+1) / 2$, that the horizon Komar-Tomimatsu mass $M_{H}$ is negative definite. While this conclusion seems surprising, we should keep in mind the well-known fact that energy is not localisable in general relativity. As demonstrated by Tomimatsu [18] and discussed in detail in [14, the total Komar mass, given by the integral over a spacelike surface at infinity

$$
M=\frac{1}{4 \pi} \oint_{\infty} D^{\nu} k^{\mu} d \Sigma_{\mu \nu}
$$

(where $k^{\mu}=\delta_{t}^{\mu}$ is the Killing vector associated with time translations), which is positive definite by the positive energy theorem, can be transformed in the Einstein-Maxwell case into a sum $M=\sum_{n} M_{n}$ over spacelike surfaces $\Sigma_{n}$ bounding the various "sources". In the present dihole case, this sum is

$$
M=2 M_{H}+M_{S}
$$


where $M_{H}$ is given by (3.18), and

$$
M_{S}=\frac{1}{8 \pi} \oint_{\Sigma_{S}}\left[g^{i j} g^{t a} \partial_{j} g_{t a}+2\left(A_{t} F^{i t}-A_{\varphi} F^{i \varphi}\right)\right] d \Sigma_{i},
$$

where $\Sigma_{S}$ is a small cylinder of radius $\xi$ surrounding the string $x=1$, $-1<y<1$, and $x^{a}=(t, \varphi)$. The individual contributions to the sum (5.12) do not necessarily have a definite sign (see e.g. the discussion in [19]), only their sum $M=\kappa m$ must be positive. In the present case, $M_{H}<0$ means that $M_{S}$ must be positive.

The necessary regularity condition (5.8) also implies that

$$
\nu(\nu-\lambda)=\frac{m^{2} b \nu}{2}+(m+2) \nu^{2}>\frac{m(m+1)}{2},
$$

which is positive definite. This means that the horizon angular velocity $\Omega_{H}$ never vanishes, and has the same sign as $\nu$, while the sign of the Komar horizon angular momentum $J_{H}$ is opposite to that of $\nu$. On the other hand, the total angular momentum $J=\kappa^{2} m(b+2 \nu)$ does not have a definite sign, and vanishes for $b=-2 \nu$, the positivity of $\bar{\mu}^{2}$ together with the necessary regularity condition (5.8) implying that the corresponding mass parameter $m$ must then lie in the range $1<m<\sqrt{2}$.

The parameter $\alpha_{H}$ associated with the conical singularity of the horizon and string can be reexpressed as

$$
\alpha_{H}=\frac{1}{k^{2}}\left(\frac{m b-2 \nu}{m b+2 \nu}\right)^{2},
$$

with

$$
k^{2}=\frac{(m+1)^{3}(m+2)}{2}>1 .
$$

It follows that, in the first regularity sector $m b / 2 \nu>1, \alpha_{H}<1$, i.e. the string tension is positive. The situation is different in the second sector $m b / 2 \nu<-1$ with the condition (5.8), which can be rewritten as

$$
-\frac{m b}{2 \nu}<\frac{(m+1)^{2}+1}{(m+1)^{2}-1}<\frac{k+1}{k-1}
$$

the last relation following from $k<(m+1)^{2}$. The conclusion is then that, in this second sector, $\alpha_{H}>1$, i.e. the string tension is negative.

Expanding the bracket in the expression (5.9) for the horizon NUT charge gives

$$
N_{H}=\frac{\kappa \alpha_{H}(m+2) \nu}{8}\left\{m z^{2}+2\left[(m+1)^{2}+1\right] z+m(2 m+3)\right\},
$$


with $z=m b / 2 \nu$. The large bracket has two negative roots

$$
z_{ \pm}=\frac{1}{m}\left[-(m+1)^{2}-1 \pm(m+1) \sqrt{m^{2}+4}\right]
$$

and is obviously positive definite in the first solution sector $z>1$, so that the sign of the horizon NUT charge is equal to that of the parameter $\nu$, and opposite to that of the horizon Komar angular momentum $J_{H}$. We can show that, in the second regular solution sector,

$$
z_{-}<-1-\frac{2}{m(m+2)}<z<-1<z_{+}
$$

so that the sign of the horizon NUT charge, and thus also of the NUT dipole $2 \kappa N_{H}$, is now equal to that of the horizon Komar angular momentum $J_{H}$.

\section{The Bonnor class of solutions}

The analysis also simplifies when the total quadrupole electric moment vanishes,

$$
\nu=0
$$

leading to

$$
d=m^{2} / 4, \quad \bar{\mu}^{2}=m^{2}\left[1-m^{2} / 4+b^{2}\right] .
$$

Then the parameter $b$ coincides with the (scaled) angular momentum to mass ratio $a$. If further $b=0$ we recover the Bonnor magnetostatic solution $a=b=0$ [8], so that we will refer to this class of solutions as the Bonnor class.

For the Bonnor class, the first equation (5.4) for the ring singularity reads

$$
\left[x^{2}-1+\delta\left(1-y^{2}\right)\right]\left[x^{2}-1+\delta\left(1-y^{2}\right)+m x+m^{2} / 2\right]=0,
$$

with

$$
\delta=1-\frac{m^{2}}{4}
$$

If $m>2$, the first bracket vanishes for $x=m / 2, y=0$, which is therefore the locus of the ring singularity. In particular, the vacuum solution of this class $\left(\bar{\mu}=0\right.$, leading to $\left.b^{2}=m^{2} / 4-1\right)$ is singular. In the opposite case $m<2$, both brackets are positive definite, so that the ring singularity is absent from the stationary region (it is hidden behind the horizon), all real values of the rotation parameter $b$ being allowed from (6.2). Note that in 
this case the ratio $|J| / M^{2}=|b| / m$ is unbounded and can be arbitrarily large, while the ratio $|\mu| / \mid J$ exceeds the Barrow-Gibbons [20] bound 1 .

The horizon angular velocity

$$
\Omega_{H}=\frac{4 b}{\kappa\left[(m+2)^{2}+4 b^{2}\right]}
$$

has the same sign as that of the total angular momentum. It is bounded above (in absolute magnitude) by

$$
\left|\Omega_{\max }\right|=\frac{1}{\kappa(m+2)}
$$

value attained for $|b|=1+m / 2$, and goes to zero both in the static limit $b \rightarrow 0$ and in the limit $b \rightarrow \infty$. Note that in this last limit $\omega-\Omega_{H}^{-1} \rightarrow 0$ everywhere (not only on the horizon). The conical singularity parameter $\alpha_{H}$ takes the value

$$
\alpha_{H}=\frac{16\left[(m+2)^{2}+4 b^{2}\right]}{(m+2)^{4}\left[(m-2)^{2}+4 b^{2}\right]} .
$$

For $b=0$ (Bonnor), $\alpha_{H}=16 /\left(m^{2}-4\right)^{2}>1$ (negative string tension) in the range $m<2$ where the ring singularity is absent [7. When the rotation parameter $b$ is turned on, $\alpha_{H}$ decreases until a critical value $b=b_{c}$ :

$$
b_{c}^{2}=\frac{m(m+2)^{2}\left(8-m^{2}\right)}{4(m+4)\left(m^{2}+4 m+8\right)}
$$

such that $\alpha_{H}\left(m, b_{c}(m)\right)=1$, i.e. the conical singularity is absent. $b_{c}$ is clearly real in the range $0<m<2$. For $b>b_{c}, \alpha_{H}$ continues to decrease towards a limiting value $16 /(m+2)^{4}$ for $|b| \rightarrow \infty$.

It is generally assumed that, except in the special case of the static Majumdar-Papapetrou linear superpositions, multi-black hole systems are unbalanced, and the force necessary to stabilize such systems is proportional to the tension $\left(1-\alpha_{S}\right) / 4$ (or $\left(1-\hat{\alpha}_{S}\right) / 4$ in the horizon co-rotating frame) of the interconnecting cosmic string(s). Indeed it has been shown 21, 22, that balance between two non-degenerate black holes cannot be achieved without intermediate conical singularities. The situation concerning rotating multiblack hole systems with degenerate horizons is less clear. It is noteworthy that there is at least a two-parameter subclass of Manko et al. solutions for which the conical singularity is absent, suggesting that these systems may be in equilibrium. We will return to this point in the concluding section. 
The values of the other horizon parameters are

$$
\begin{aligned}
M_{H} & =\frac{4 \kappa m b^{2}\left[(m-2)^{2}\left(m^{2}+2 m+4\right)+4 b^{2}\left(m^{2}-2 m+4\right)\right]}{(m+2)^{2}\left[(m-2)^{2}+4 b^{2}\right]^{2}}, \\
N_{H} & =\frac{\kappa \alpha_{H} m(m+2)^{2} b}{8}, \quad P_{H}=\frac{\kappa \bar{\mu}\left(4-m^{2}-4 b^{2}\right)}{(m+2)\left[(m-2)^{2}+4 b^{2}\right]}, \\
Q_{H} & =\frac{8 \kappa \bar{\mu} b}{(m+2)\left[(m-2)^{2}+4 b^{2}\right]}, \quad \phi_{H}=\frac{\kappa \bar{\mu}}{m} \Omega_{H}
\end{aligned}
$$

(the value of the horizon angular momentum $J_{H}$ can be retrieved from the horizon Smarr formula (3.21) ). The horizon Komar mass is positive definite.

The limiting case $\underline{b=0}$ with $m<2$ corresponds to the Bonnor solution. Eq. (6.9) shows that $M_{H}=0$ in this case. The fact that the horizon Komar mass of the Bonnor magnetostatic solution vanishes, which does not seem to have been pointed out previously, is a direct consequence of the Smarr relation for a static, electrically neutral field configuration with degenerate horizons. It then follows from (5.12) that the mass of the Bonnor dihole must be equal to the string Komar mass. This is checked in the Appendix by an independent direct evaluation of (5.13). The only non-zero horizon observables of the Bonnor dihole are their magnetic charges $\pm P_{H}$, with

$$
P_{H}=\frac{\kappa \bar{\mu}}{2-m},
$$

the dipole moment $2 \kappa P_{H}$ accounting partly for the total magnetic moment $\mu=\kappa^{2} \bar{\mu}$, with which it coincides in the limit $m \rightarrow 0$.

\subsection{Large distance limit}

The large distance limit can be defined as the limit when the distance $2 \kappa$ between the two horizons becomes very large, $\kappa \rightarrow \infty$, while the total mass $M=\kappa m$ is held fixed, i.e. $m \rightarrow 0$. In this limit the ratio $|J| / M^{2}=|b| / m$ can be arbitrary large, unless $b$ goes also to zero. The horizon angular velocity and the string tension

$$
\Omega_{H} \sim \frac{b}{\kappa\left(1+b^{2}\right)}, \quad \frac{1-\alpha_{H}}{4} \sim \frac{M b^{2}}{2 \kappa\left(1+b^{2}\right)}
$$

both go to zero as $1 / \kappa$. An exception is the Bonnor static dihole $b=0$, for which $\Omega_{H}=0$ but $\left(1-\alpha_{H}\right) / 4 \sim-M^{2} / 8 \kappa^{2}[7]$. 
The large distance values of the other horizon observables are

$$
\begin{aligned}
M_{H} & \sim \frac{M b^{2}}{1+b^{2}}, \quad N_{H} \sim \frac{M b}{2} \\
Q_{H} & \sim \varepsilon \frac{M b}{\sqrt{1+b^{2}}}, \quad P_{H} \sim \varepsilon \frac{M\left(1-b^{2}\right)}{2 \sqrt{1+b^{2}}}
\end{aligned}
$$

$(\varepsilon= \pm 1)$ and 4

$$
J_{H} \sim \frac{M^{2} b\left(1-b^{2}\right)}{2\left(1+b^{2}\right)} \sim Q_{H} P_{H} .
$$

This last relation shows that in this limit the dyonic black hole angular momentum $J_{H}$ is not an inertial effect (the horizon angular velocity goes to zero), but is purely electromagnetic. Note also that for fixed $b$ the total angular momentum

$$
J=\kappa M b \sim 2 \kappa N_{H} \gg J_{H}
$$

is due essentially to the NUT dipole. If the parameter $b$ also goes to zero (slowly rotating Bonnor dihole), then as in the static Bonnor case all the horizon observables go to zero, except for the horizon magnetic charge $P_{H} \sim$ $\varepsilon M / 2$, leading to the magnetic dipole $\mu \sim 2 \kappa P_{H}$.

An interesting special case is $|b|=1$. Then,

$$
M_{H} \sim\left|N_{H}\right| \sim \frac{M}{2}, \quad J_{H} \sim 0, \quad\left|Q_{H}\right| \sim \frac{M}{\sqrt{2}}, \quad P_{H} \sim 0 .
$$

In this case the string mass $M_{S}=M-2 M_{H}$ goes to zero, while the horizon observables satisfy the static extremality condition

$$
M_{H}^{2}+N_{H}^{2} \sim \frac{M^{2}}{2} \sim Q_{H}^{2}+P_{H}^{2}
$$

\section{The static class of solutions}

The horizon angular velocity vanishes, so that the constituent black holes become static, for

$$
\lambda=\nu \quad \Leftrightarrow \quad b=-\frac{4 \nu \delta}{m^{2}}
$$

which leads to

$$
\delta=1+\nu^{2}-\frac{m^{2}}{4} \geq 0
$$

\footnotetext{
${ }^{4}$ To evaluate the limit of $J_{H}$ one must expand $M_{H}, Q_{H}$ and $\phi_{H}$ to order $\mathrm{O}\left(\mathrm{m}^{2}\right)$, as the terms of order $\mathrm{O}(m)$ cancel in (3.21).
} 
this last restriction following from the condition

$$
\bar{\mu}^{2}=\frac{16 \delta\left(\nu^{2}+d^{2}\right)}{m^{2}} \geq 0 .
$$

If further $\nu=0$ we recover again the Bonnor magnetostatic solution $a=b=$ $0\left(\lambda=\nu=0, d=m^{2} / 4\right)$. Another special case is $\delta=0\left(\nu^{2}=m^{2} / 4-1\right)$, leading to the rotating TS2 vacuum solution [16].

We shall refer to this class of solutions with non-rotating constituent black holes as the "static" class. Despite this appellation, the solutions are generically non-static, with net total angular momentum $J=\kappa^{2} m a$, where

$$
a=\frac{2 \nu\left(m^{2}-2 \delta\right)}{m^{2}} .
$$

This angular momentum is due partly to the dipole moment of the two opposite NUT charges (4.10) carried by the black hole horizons, and partly to the string angular momentum. The two exactly balance, so that the rotation parameter (7.4) vanishes, $a=0$, if $\delta=m^{2} / 2$, corresponding to

$$
4 \nu^{2}=3 m^{2}-4, \quad \bar{\mu}^{2}=2 m^{2}\left(m^{2}-1\right) \quad(m>2 / \sqrt{3}) .
$$

This stationary magnetized solution, with non-rotating horizons and vanishing total angular momentum, reduces for $\nu=0$ to the static Bonnor solution with the special value $m=2 / \sqrt{3}$.

For the static class,

$$
\operatorname{Re}(U+W) \equiv \zeta(\zeta+m x+2 d)+\nu\left[m b x-\nu\left(1+y^{2}\right)\right]\left(1-y^{2}\right) .
$$

While for the Bonnor solution this is well-known to be positive definite for $|m|<2$, in the general case there does not seem to be a parameter range where $\operatorname{Re}(U+W)$ is positive definite. For $y=0$ and $x=1$, one obtains

$$
\operatorname{Re}(U+W)(1,0) \equiv \frac{m+4}{m}\left[-\delta^{2}+\delta+\frac{m\left(4-m^{2}\right)}{4(m+4)}\right] .
$$

The discriminant of the trinomial in $\delta$ inside brackets

$$
\Delta=\frac{(m+1)\left(-m^{2}+m+4\right)}{m+4}
$$

is positive for $m<m_{0}=(1+\sqrt{17}) / 2 \simeq 2.56$, in which case (7.7) has two roots $\delta_{ \pm}=(1 \pm \sqrt{\Delta}) / 2$. So, given that $\operatorname{Re}(U+W)$ is positive for large $x$, a necessary condition for the absence of equatorial ring singularity is

$$
m<m_{0}, \quad \delta_{-}<\delta<\delta_{+} .
$$


The TS2 solution $\left(\delta=0<\delta_{-}\right)$is well-known to present a ring singularity. For the static subclass $a=0$, the necessary condition $\operatorname{Re}(U+W)(1,0)>0$ reduces to

$$
(m+1)\left(-m^{3}-3 m^{2}+4 m+4\right)>0,
$$

leading to

$$
2 / \sqrt{3} \simeq 1.15 \leq m<m_{1} \simeq 1.49 \quad(a=0) .
$$

Because the horizon angular velocity vanishes, the conical singularity parameter is simply $\alpha_{H}=\hat{\alpha}_{S}=\alpha_{S}=1 /\left(\delta^{2}+\nu^{2}\right)$. For the $a=0$ subclass, this gives

$$
\alpha_{H}=\frac{4}{\left(m^{2}-1\right)\left(m^{2}+4\right)} \quad(a=0) .
$$

This is bounded above by $\alpha_{\max }=\alpha(2 / \sqrt{3})=9 / 4$, and goes to zero for $m \rightarrow \infty$. So there is a critical value

$$
m_{c}=[(-3+\sqrt{41}) / 2]^{1 / 2} \simeq 1.30 \quad(a=0)
$$

such that the conical singularity is absent, $\alpha_{H}\left(m_{c}\right)=1$. This value of $m_{c}$ satisfies the necessary regularity condition (7.9).

Although, strictly speaking, the Tomimatsu formulas we have used to compute the various horizon parameters break down for $\Omega_{H}=0$, we can nevertheless use here our results (3.17) and (3.20), together with (3.14) and (4.10), in the limit $\Omega_{H} \rightarrow 0(\lambda \rightarrow \nu)$. We give here the results only for the subclass $\underline{a=0}$ :

$$
\begin{aligned}
N_{H} & =-\frac{2 \kappa \nu m(m+1)}{(m-1)\left(m^{2}+4\right)}, \quad P_{H}=-\frac{\kappa \bar{\mu}\left[2 m^{3}-3 m^{2}+4 m-4\right]}{2(m-1)^{2}\left(m^{2}+4\right)}, \\
Q_{H} & =--\frac{\kappa \bar{\mu} \nu(2-m)}{(m-1)^{2}\left(m^{2}+4\right)}, \quad \phi_{H}=-\frac{\bar{\mu} \nu}{2 m\left(m^{2}-1\right)} \quad(a=0(7 . .12)
\end{aligned}
$$

The horizon mass is given by (3.20), which can be written as $M_{H}=M_{g}+M_{e}$, where the terms $M_{g}$ and $M_{e}$ coming from the two terms in the (modified) Tomimatsu formula (3.18) can be thought of, loosely speaking, as the "gravitational" and "electromagnetic" contributions to the horizon KomarTomimatsu mass. For consistency, we must check the Smarr formula (3.21), which for $\Omega_{H}=0$ reduces to $M_{H}=\phi_{H} Q_{H}$. We obtain for $a=0$ :

$$
M_{g}=\frac{\kappa m^{2}(2-m)^{2}}{2(m-1)\left(m^{2}+4\right)}, \quad M_{e}=\frac{\kappa m(2-m)\left[2 m^{3}-3 m^{2}+4 m-4\right]}{4(m-1)^{2}\left(m^{2}+4\right)},
$$


leading to

$$
M_{H}=\frac{\kappa m(2-m)\left(3 m^{2}-4\right)}{4(m-1)^{2}\left(m^{2}+4\right)}=\phi_{H} Q_{H} \quad(a=0) .
$$

This is positive definite in the regularity range (7.9). One can check that the string mass $M_{S}=M-2 M_{H}$ is also positive in this range.

\section{Summary and discussion}

We have analyzed the four-parameter class of asymptotically flat magnetized solutions to the Einstein-Maxwell equations constructed in [12], and shown that these represent systems of two co-rotating extreme black holes with equal masses and electric charges, and opposite magnetic and NUT charges, connected by a cosmic string. A special two-parameter subclass of solutions, without ring singularity, was previously discussed in [9]. We have discussed here in some detail several other three-parameter subclasses, and determined in each case the parameter domain in which the ring singularity is absent. For the "neutral" class (electrically neutral constituents), there are two regularity sectors. In the first sector the string tension is positive, while it is negative in the second sector. In both sectors, the horizon Komar mass is negative. For the Bonnor class, there is only one regularity sector, with varying string tension, which vanishes in a two-parameter subclass. The horizon Komar mass is always positive, except for the static Bonnor solution itself, where it vanishes. We have also considered for this class the large distance limit $\kappa \rightarrow \infty, m \rightarrow 0$. Finally, for the "static" class (rotating solutions with non-rotating constituents), there is also a single regularity sector. We have discussed in more detail the two-parameter subclass such that the total angular momentum vanishes, and have found that for this subclass the horizon mass, as well as the string mass, are positive. Again, the string tension vanishes in a one-parameter family of this subclass.

A remarkable finding is the existence of rotating systems of extreme black holes with vanishing tension of the interconnecting string. This calls for two observations. In the presence of NUT charges, this string is also a Misner string, with spin $\hat{\omega}_{S}$ (in the horizon co-rotating frame) proportional to the NUT charge, see (4.9). If the horizons are rotating, then the string tension in the global frame (that of an observer at spatial infinity) is different from the string tension in the local horizon co-rotating frame, as follows from the last equation (4.7). So our statement that the string tension vanishes

is ambiguous. Actually, it is valid in the horizon co-rotating frame, where 
$\hat{\alpha}_{S}=\alpha_{H}=1$ means that the constituent black holes feel no tension, so that their horizon is smooth. Yet the asymptotic observer can in principle measure a tension $\left(1-\alpha_{S}\right) / 4$, with

$$
\alpha_{S}=\frac{\alpha_{H}}{1-4 N_{H} \Omega_{H}} .
$$

For the Bonnor subclass $\left(\kappa, m, b_{c}(m)\right)$ (with $b_{c}$ given by (6.8)), the horizon angular velocity and NUT charge are of the same sign (see (6.5) and (6.9)), so that $\alpha_{S}>1$, corresponding to a negative observed string tension, to compensate an observed attraction between the two black holes. On the other hand, our statement that there is a one-parameter (the scale $\kappa$ ) family of solutions in the subclass $a=0$ of the static class without a conical singularity is unambiguous, as the horizons are non-rotating.

The second observation is that for axisymmetric solutions of Einstein's equations, written in the Weyl form

$$
d s^{2}=-F(d t-\omega d \varphi)^{2}+F^{-1}\left[e^{2 k}\left(d \rho^{2}+d z^{2}\right)+\rho^{2} d \varphi^{2}\right],
$$

there are two regularity conditions on the symmetry axis $\rho=0, k(0, z)=$ 0 , and $\omega(0, z)=0$. These are satisfied by construction on the two semiinfinite portions $y= \pm 1, x>1$ of the axis, but not, generically, on the interconnecting string $x=1,-1<y<1$, where they translate into $\alpha_{S}=1$, $\omega_{S}=0$, or $\hat{\alpha}_{S}=1$ and $\hat{\omega}_{S}=0$ in the horizon co-rotating frame. In other words, in the unambiguous 'static' case with vanishing string tension, there remains a Misner string singularity. We expect that, contrary to a cosmic string, where geodesics terminate, this Misner string will be transparent to geodesic motion, as shown in [23] in the cases of the Taub-NUT metric and of the dyonic Reissner-Nordström-NUT metric. However there is always, in the vicinity of a Misner string, an 'acausal' region containing closed timelike curves (CTC). We have argued in [23, 24] that the existence of such a region where $g_{\varphi \varphi}=0$ does not necessarily lead to observable violations of causality. While this matter clearly deserves further investigation, we feel that the possibility of spacetimes with finite-length Misner strings and the associated compact CTC regions should be left open.

The preliminary analysis reported here should be extended to a more systematic investigation of this four-parameter class of solutions, starting with the determination in the general case of the parameter domain for which the ring singularity is absent. 


\section{Acknowledgments}

I warmly thank Dmitry Gal'tsov for a critical reading of the manuscript and useful suggestions.

\section{Appendix: Computation of the Bonnor string mass}

This is given by (5.13), which reads in the case of the Bonnor magnetostatic solution

$$
\left.M_{S}=\lim _{(\xi \rightarrow 0)} \frac{1}{4} \int_{-1}^{+1}\left[g^{\xi \xi} g^{t t} \partial_{\xi} g_{t t}-2 A_{\varphi} F^{\xi \varphi}\right)\right] \sqrt{|g|} d y
$$

with $\xi^{2} \equiv x^{2}-1$. The first, purely gravitational term does not contribute in the limit $\xi \rightarrow 0$ because $g_{t t}=$ constant $+\mathrm{O}\left(\xi^{2}\right)$. From (2.8) and (2.9) with $b=\nu=0$, we obtain

$$
A_{\varphi}=\frac{\kappa \bar{\mu}\left(1-y^{2}\right)(2 x+m)}{2\left[x^{2}-1+\delta\left(1-y^{2}\right)\right]}
$$

leading to

$$
F_{\xi \varphi}=-\frac{\kappa \bar{\mu}\left(\tau^{2}+y^{2}\right)}{\delta\left(1-y^{2}\right)} \xi+\mathrm{O}\left(\xi^{3}\right)
$$

where we have put

$$
\tau^{2} \equiv \frac{m+2-\delta}{\delta}=\frac{m+2}{2-m}
$$

Using the string metric (4.4) for $b=\nu=0$, where

$$
\Sigma_{S}=\delta^{4}\left(1-y^{2}\right)^{2}\left(\tau^{2}-y^{2}\right)^{2}+\mathrm{O}\left(\xi^{2}\right)
$$

we then obtain on the string $\xi=0$

$$
A_{\varphi}=\frac{\kappa \bar{\mu}(m+2)}{2 \delta}, \quad \sqrt{|g|} F^{\xi \varphi}=-\frac{\bar{\mu}}{\delta} \frac{\tau^{2}+y^{2}}{\left(\tau^{2}-y^{2}\right)^{2}},
$$

leading to

$$
M_{S}=\frac{2 \kappa m^{2}}{2-m} \frac{1}{\tau^{2}-1}=\kappa m=M .
$$




\section{References}

[1] R. Bach and H. Weyl, Math. Zeits. 13 (1922) 134.

[2] W. Israel and K.A. Khan, Nuovo Cimento 33 (1964) 331.

[3] A. Papapetrou, Proc. Roy. Irish Acad. A 51 (1947) 191; S.D. Majumdar, Phys. Rev. 72 (1947) 390.

[4] G. Weinstein, Commun. Part. Diff. $\quad$ Eq. $21 \quad$ (1996) 1389 arXiv:gr-qc/0001081.

[5] J.B. Griffiths and J. Podolsky, Exact space-times in Einstein's General Relativity, CUP, 2009.

[6] I. Cabrera-Munguia, V.E. Ceron, L.A. López and Omar Pedraza, Phys. Lett. B 772 (2017) 10 arXiv:1702.02209]; V.S. Manko and E. Ruiz, Phys. Rev. D 96 (2017) 104016 arXiv:1702.05802.

[7] R. Emparan, Phys. Rev. D 61 (2000) 104009 [arXiv:hep-th/9906160].

[8] W.B. Bonnor, Z. Phys. 190 (1966) 444.

[9] G. Clément and D. Gal'tsov, Phys. Lett. B $\mathbf{7 7 1}$ (2017) 457 arXiv:1705.08017]; "Stationary binary black holes without naked ring singularity" arXiv:1806.11193.

[10] G. Clément, Phys. Rev. D 57 (1998) 4885 arXiv:gr-qc/9710109.

[11] N.R. Sibgatullin: Oscillations and Waves in Strong Gravitational and Electromagnetic Fields (Nauka, Moscow, 1984; English translation: Springer-Verlag, Berlin, 1991).

[12] V.S. Manko, E.W. Mielke and J.D. Sanabria-Gomez, Phys. Rev. D 61 (2000) 081501 arXiv:gr-qc/0001081.

[13] V.S. Manko, J.D. Sanabria-Gomez and O.V. Manko, Phys. Rev. D 62 (2000) 044048.

[14] G. Clément and D. Gal'tsov, Phys. Lett. B 773 (2017) 290 arXiv:1707.01332.

[15] D.M. Zipoy, J. Math. Phys. 7 (1966) 1137; B. H. Voorhees, Phys. Rev. D 2 (1970) 2119. 
[16] A. Tomimatsu and H. Sato, Phys. Rev. Lett. 29 (1972) 1344; Progr. Theor. Phys. 50 (1973) 95.

[17] H. Kodama and W. Hikida, Class. Quantum Grav. 20, 5121 (2003) [arXiv: gr-qc/0304064].

[18] A. Tomimatsu, Progr. Theor. Phys. 72 (1984) 73.

[19] A. Tomimatsu, Prog. Theor. Phys. 70 (1983) 385.

[20] J.D. Barrow and G.W. Gibbons, Phys. Rev. D 95 (2017) 064040 arXiv:1701.06343.

[21] W. Dietz and C. Hoenselaers, Ann. Phys. 165 (1985) 319.

[22] J. Sod-Hoffs and E.D. Rodchenko, Class. Quantum Grav. 24 (2007) 4617 arXiv:0705.3973.

[23] G. Clément, D. Gal'tsov and M. Guenouche, Phys. Lett. B 750 (2015) 591 arXiv:1508.07622]; Phys. Rev. D 93 (2016) 024048 arXiv:1509.07854.

[24] G. Clément and M. Guenouche, Gen. Rel. Grav. 50 (2018) 60 arXiv:1606.08457. 